\title{
Non-Singularity Conditions for Two Z-Matrix Types
}

\author{
Shinji Miura \\ Independent, Gifu, Japan \\ Email: geppa_gifu@yahoo.co.jp
}

Received 23 April 2014; revised 23 May 2014; accepted 30 May 2014

Copyright (C 2014 by author and Scientific Research Publishing Inc.

This work is licensed under the Creative Commons Attribution International License (CC BY). http://creativecommons.org/licenses/by/4.0/

(c) (i) Open Access

\section{Abstract}

A real square matrix whose non-diagonal elements are non-positive is called a Z-matrix. This paper shows a necessary and sufficient condition for non-singularity of two types of Z-matrices. The first is for the Z-matrix whose row sums are all non-negative. The non-singularity condition for this matrix is that at least one positive row sum exists in any principal submatrix of the matrix. The second is for the Z-matrix $A$ which satisfies $A x \geq 0$ where $\exists x>0$. Let $a_{i j}$ be the ith row and the $j$ th column element of $A$, and $x_{j}$ be the $j$ th element of $x$. Let $F$ be a subset of $N=\{1,2, \cdots, n\}$ which is not empty, and $G$ be the complement of $F$ if $F$ is a proper subset. The non-singularity condition for this matrix is $\exists i \in F$ such that $\sum_{j \in N} a_{i j} x_{j}>0$ or $\exists i \in F, \exists j \in G$ such that $a_{i j}<0$ for $\forall F \subseteq N$. Robert Beauwens and Michael Neumann previously presented conditions similar to these conditions. In this paper, we present a different proof and show that these conditions can be also derived from theirs.

\section{Keywords}

Z-Matrix, M-Matrix, Non-Negative Matrix, Diagonal Dominance

\section{Introduction}

A real square matrix whose non-diagonal elements are non-positive is called a Z-matrix. The purpose of this paper is to show a necessary and sufficient condition for non-singularity of two types of Z-matrices.

The first is the Z-matrix whose row sums are all non-negative. In this paper, we denote this as a Non-negative Sums Z-matrix (NSZ-matrix).

The second is the Z-matrix $A$ which satisfies $A x \geq 0$ where $\exists x>0$. In this paper, we denote this as a 
Non-negative Product Z-matrix (NPZ-matrix).

The following relation exists between these matrices.

Theorem 1.1 An NSZ-matrix is equivalent to an NPZ-matrix where all elements of $x$ are the same number.

Proof. Let $N=\{1,2, \cdots, n\}$ be a set of numbers, and $x$ be a positive vector with all elements equal to the same number $x^{*}$.

If $A=\left(a_{i j}\right)$ is an NSZ-matrix, the $i$ th element of $A x$ is $\left(\sum_{j \in N} a_{i j}\right) x^{*} . \sum_{j \in N} a_{i j} \geq 0$ for $\forall i \in N$ because $A$ is an NSZ-matrix. Also, $x^{*}>0$ from the premise. Hence, $\left(\sum_{j \in N} a_{i j}\right) x^{*} \geq 0$ for $\forall i \in N$ is satisfied. Therefore, $A$ is an NPZ-matrix.

Conversely, consider that $A=\left(a_{i j}\right)$ is an NPZ-matrix which satisfies $\left(\sum_{j \in N} a_{i j}\right) x^{*} \geq 0$ for $\forall i \in N$ where $\exists x^{*}>0$. If we divide both sides of $\left(\sum_{j \in N} a_{i j}\right) x^{*} \geq 0$ by $x^{*}>0$, we obtain $\sum_{j \in N} a_{i j} \geq 0$ for $\forall i \in N$. Thus, $A$ is an NSZ-matrix. [Q. E. D.]

As Theorem 1.1 shows, the NSZ-matrix is a type of the NPZ-matrix. Therefore, if we can find a necessary and sufficient condition for non-singularity of the NPZ-matrix, we find the $(a)$ necessary and sufficient condition for non-singularity of the NSZ-matrix automatically. However, we will prove the latter condition first, and then address the former condition.

We first state the basic propositions of linear algebra used in this paper.

The determinant of a square matrix $A$ is $\operatorname{denoted} \operatorname{det} A$ in this paper.

Theorem 1.2 Let $A=\left(a_{i j}\right)$ be a Z-matrix. Take a real number $\rho$ which is equal to or more than all diagonal elements and construct the matrix $B=\rho E-A$ where $E$ refers to the unit matrix. $B$ is a non-negative matrix.

Proof. The non-diagonal elements of $B=\left(b_{i j}\right)$ are $b_{i j}=-a_{i j}$. As $A$ is a Z-matrix, $b_{i j}=-a_{i j} \geq 0$ for $\forall i \neq j$. On the other hand, the diagonal elements of $B$ are $b_{i i}=\rho-a_{i i}$. Since $\rho \geq a_{i i}$ for $\forall i \in N$ by the premise, $b_{i i} \geq 0$ for $\forall i \in N$. Therefore, $\forall b_{i j} \geq 0$. [Q. E. D.]

Theorem 1.3 A non-negative square matrix $B$ always has a non-negative eigenvalue. Let $\lambda(B)$ be the maximum non-negative eigenvalue of $B$. Then there exists a non-negative eigenvector corresponding to $\lambda(B)$.

If a Z-matrix $A=\rho E-B$ satisfies $\lambda(B) \leq \rho, A$ is called an M-matrix ${ }^{1}$.

Theorem 1.4 An M-matrix $A$ is non-singular if and only if $\lambda(B)<\rho$. In this case, $\rho>0$ and all elements of the inverse of an M-matrix are non-negative. In particular, all diagonal elements of the inverse are equal to or more than $1 / \rho^{2}$.

Proof. As Theorems 1.3 and 1.4 are well known, we entrust the proof to another work ${ }^{3}$. However, as it receives less attention that diagonal elements of $A^{-1}$ are $1 / \rho$ or more, we confirm this aspect.

Regarding the inverse of an M-matrix $A=\rho E-B, A^{-1}=E / \rho+\sum_{k=1}^{\infty}\left(B^{k} / \rho^{k+1}\right)$ is satisfied ${ }^{4}$. Thus, if we set $A=\left(a_{i j}\right)$ and $B^{k}=\left\{b_{i j}^{(k)}\right\}, a_{i i}=1 / \rho+\sum_{k=1}^{\infty}\left\{b_{i i}^{(k)} / \rho^{k+1}\right\}$ is satisfied. As $B$ is a non-negative matrix and $\rho>0, \quad b_{i i}^{(k)} / \rho^{k+1} \geq 0$ for $\forall k \geq 1$. Therefore, we can obtain $a_{i i}=1 / \rho+\sum_{k=1}^{\infty}\left\{b_{i i}^{(k)} / \rho^{k+1}\right\} \geq 1 / \rho$. [Q. E. D.]

Theorem 1.5 If the row sums of a square matrix $A$ are all zeroes, det $A=0$.

Proof. Let $k_{j}$ be the $j$ th column vector of $A$. We construct the linear combination $\lambda_{1} k_{1}+\lambda_{2} k_{2}+\cdots+\lambda_{n} k_{n}$. If the row sums of $A$ are all zeroes, $\lambda_{1} k_{1}+\lambda_{2} k_{2}+\cdots+\lambda_{n} k_{n}=0$ when $\lambda_{1}=\lambda_{2}=\cdots=\lambda_{n}=1$. Therefore, $k_{1}, k_{2}, \cdots, k_{n}$ are linearly dependent. The determinant of a matrix whose column vectors are linearly dependent is zero ${ }^{5}$. [Q. E. D.]

If $B=P^{-1} A P$ holds for the square matrices $A$ and $B$ and a non-singular matrix $P, A$ and $B$ are called similar to each other.

\footnotetext{
${ }^{1}$ This definition is that given by Berman \& Plemmons [1] p. 133. Alexander Ostrowski, who used the concept M-matrix first, gave a different definition for M-matrix. Cf. Ostrowski [2] p. 69, Berman \& Plemmons [1] p. 161. The definition of M-matrix given in Varga [3] is also different. Cf. Varga [3] p. 91.

${ }^{2}$ Theorems 1.3 and 1.4 are often called Frobenius theorem after their discoverer, Georg Frobenius.

${ }^{3}$ Cf. Berman \& Plemmons [1] pp. 6-7, p. 26, Nikaido [4] pp. 101-102, Varga [3] p. 51, p. 89.

${ }^{4}$ Cf. Nikaido [4] p. 96, Varga [3] p. 89.

${ }^{5}$ Cf. DeFranza \& Gabliardi [5] pp. 118-119.
} 
Theorem 1.6 When two matrices are similar, if one matrix is non-singular, the other is also non-singular.

Theorem 1.7 Similar matrices have the same eigenvalue ${ }^{6}$.

Here, we define the notation for submatrices in this paper.

Let $N=\{1,2, \cdots, n\}$ be a set of number of rows and columns of a square matrix $A$ and let $F$ be a subset of $N$ which is not empty. $A_{F F}$ refers to a submatrix of $A$ whose row and column elements belong to $F$. When $F$ is a proper subset, we define $G$ as the complement of $F$. $A_{F G}$ refers to a submatrix of $A$ whose row elements belong to $F$ and column elements belong to $G$. Similarly, $A_{G F}$ refers to a submatrix of $A$ whose row elements belong to $G$ and column elements belong to $F$, and $A_{G G}$ refers to a submatrix of $A$ whose row and column elements belong to $G$. Clearly, $A_{F F}$ and $A_{G G}$ are principal submatrices. $A_{N N}$ is $A$ itself.

Based on the above, we confirm the following basic proposition.

Theorem 1.8 If $A_{F G}$ is a zero matrix, $\operatorname{det} A=\left(\operatorname{det} A_{F F}\right)\left(\operatorname{det} A_{G G}\right)^{7}$.

\section{A Necessary and Sufficient Condition for Non-Singularity of the Z-Matrix Whose Row Sums Are All Non-Negative}

In this section, we discuss the non-singularity of the NSZ-matrix. We reconfirm that the NSZ-matrix is defined as the Z-matrix whose row sums are all non-negative. $A=\left(a_{i j}\right)$ denotes an NSZ-matrix in this section.

Theorem 2.1 An NSZ-matrix is an M-matrix .

Proof. Take an NSZ-matrix $A$ and a real number $\rho$ which is equal to or more than all diagonal element, and construct the matrix $B=\rho E-A . B$ is a non-negative matrix from Theorem 1.2. Let $b_{i j}$ be the $i$ th row and the jth column element of $B$ and let $x_{j}$ be the jth element of a non-negative eigenvector of $B$ corresponding to $\lambda(B)$. Moreover, let $x_{k}$ be a maximum of $x_{j}$. Incidentally, multiple candidates of $k$ may exist. In that case, one can choose any $k$ of these. From the definition of eigenvalue and eigenvector,

$\lambda(B) x_{k}=\sum_{j \in N} b_{k j} x_{j}$ is satisfied. On the other hand, $x_{j} \leq x_{k}$ for $\forall j \in N$ because $x_{k}$ is a maximum of $x_{j}$. $\forall b_{i j} \geq 0$ because $B$ is a non-negative matrix. From these conditions, $b_{k j} x_{j} \leq b_{k j} x_{k}$ is satisfied generally. Thus, $\lambda(B) x_{k}=\sum_{j \in N} b_{k j} x_{j} \leq\left(\sum_{j \in N} b_{k j}\right) x_{k}$.

Here, we confirm $x_{k}>0$. Note that $x_{j} \geq 0$ because it is an element of non-negative eigenvector. Therefore, if $x_{k}$ is zero, $\forall x_{j}=0$ because $x_{k}$ is the maximum of $x_{j}$. However, the eigenvector is not a zero vector from its definition. Hence, $x_{k}>0$.

Based on the above, we divide both sides of the formula $\lambda(B) x_{k} \leq\left(\sum_{j \in N} b_{k j}\right) x_{k}$ by $x_{k}>0$. Then, we can derive $\lambda(B) \leq \sum_{j \in N} b_{k j}$. Note that $\rho-\sum_{j \in N} b_{i j} \geq 0$ for $\forall i \in N$ because $A=\rho E-B$ is an NSZ-matrix. Then, $\lambda(B) \leq \sum_{j \in N} b_{k j} \leq \rho$. It is obvious that $A$ satisfies the definition of an M-matrix. [Q. E. D.]

Theorem 2.2 An NSZ-matrix is non-singular if and only if it is a non-singular M-matrix.

Proof. It is obvious that an NSZ-matrix is non-singular if it is a non-singular M-matrix. Conversely, if an NSZ-matrix is non-singular, it is a non-singular M-matrix by Theorem 2.1. [Q. E. D.]

Considering Theorem 2.2, we see that finding a necessary and sufficient condition for non-singularity of the NSZ-matrix equates to finding a condition that it is a non-singular M-matrix. We will show this.

Theorem 2.3 All row sums of any principal submatrix of an NSZ-matrix are non-negative.

Proof. Regarding A itself, this is obvious because of the definition of the NSZ-matrix. In the following, we show a proof for principal submatrices which are not $A$ itself.

Let $F$ be a proper subset of $N$ and $G$ be the complement of $F$. As all row sums of $A$ are non-negative, $\sum_{j \in N} a_{i j}=\sum_{j \in F} a_{i j}+\sum_{j \in G} a_{i j} \geq 0$ holds for $\forall i \in F$. Hence, $\sum_{j \in F} a_{i j} \geq-\sum_{j \in G} a_{i j}$. Because $G$ is the complement of $F$, it is clear that $a_{i j}$ for $\forall i \in F, \forall j \in G$ are non-diagonal elements of the Z-matrix $A$. Therefore, all of these are non-positive. Then, $\sum_{j \in F} a_{i j} \geq-\sum_{j \in G} a_{i j} \geq 0$ for $\forall i \in F$ is true. [Q. E. D.]

Theorem 2.4 If there exists at least one principal submatrix of an NSZ-matrix whose row sums are all zeroes,

${ }^{6}$ Cf. Anton \& Rorres [6] pp. 305-306 for Theorems 1.6 and 1.7.

${ }^{7}$ Cf. Bretscher [7] p. 258.

${ }^{8}$ This theorem is stated in Plemmons [8] p. 248. 
the matrix is singular.

Proof. If all row sums of $A$ itself, which is one of the principal submatrices of an NSZ-matrix, are zeroes, the proposition is derived from Theorem 1.5 immediately. In the following, we show a proof for principal submatrices which are not $A$ itself.

Choose $F \neq \phi$ which is a proper subset of $N$, where the row sums of $A_{F F}$ are all zeroes. $G$, which is the complement of $F$, is also not empty.

Based on this, we will confirm that $A_{F G}$ is a zero matrix. From the definition of an NSZ-matrix, $\sum_{j \in N} a_{i j}=\sum_{j \in F} a_{i j}+\sum_{j \in G} a_{i j} \geq 0$ holds for $\forall i \in F$. Then, $\sum_{j \in G} a_{i j} \geq 0$ for $\forall i \in F$ are obtained because $\sum_{j \in F} a_{i j}=0$ for $\forall i \in F$ as defined. However, $a_{i j} \leq 0$ for $\forall i \in F, \forall j \in G$ because $A$ is a Z-matrix. For these two propositions to be compatible, $a_{i j}=0$ must hold for $\forall i \in F, \forall j \in G$. Therefore, $A_{F G}$ is a zero matrix.

Then, $\operatorname{det} A=\left(\operatorname{det} A_{F F}\right)\left(\operatorname{det} A_{G G}\right)$ holds by Theorem1.8. Because all row sums of $A_{F F}$ are zeroes, $\operatorname{det} A_{F F}=0$ by Theorem 1.5. Thus, $\operatorname{det} A=0$. [Q. E. D.]

Theorem 2.5 If an NSZ-matrix is non-singular, at least one positive row sum exists in any principal submatrix of the matrix.

Proof. Due to the contraposition of Theorem 2.4, if an NSZ-matrix is non-singular, there does not exist a principal submatrix whose row sums are all zeroes. Then, by Theorem 2.3, at least one positive row sum exists in any principal submatrix of the matrix. [Q. E. D.]

As a result of Theorem 2.5, a necessary condition for non-singularity of the NSZ-matrix is shown. We now prove this is also a sufficient condition.

Theorem 2.6 If at least one positive row sum exists in any principal submatrix of an NSZ-matrix, the matrix is a non-singular M-matrix.

For the proof of Theorem 2.6, we have to use inference. In the following section, we will set $A$ as an NSZ-matrix which has at least one positive row sum in any principal submatrix. Moreover, we take a real number $\rho$ which is equal to or more than all diagonal elements and construct the matrix $B=\rho E-A$. Note that, from Theorem 1.2, $B$ is a non-negative matrix. We now prove the following Lemmas.

Lemma 2.7 Any row sum of all principal submatrices of $B$ is equal to or less than $\rho$.

Proof. It is obvious that $\sum_{j \in F} b_{i j}=\rho-\sum_{j \in F} a_{i j}$ for $\forall i \in F$ from the definition of $B . \sum_{j \in F} a_{i j} \geq 0$ for $\forall i \in F$ by Theorem 2.3. Thus, $\rho-\sum_{j \in F} a_{i j} \leq \rho$. Therefore, $\sum_{j \in F} b_{i j} \leq \rho$ for $\forall i \in F$. [Q. E. D.]

Lemma 2.8 Any principal submatrix of $B$ has at least one row sum which is less than $\rho$.

Proof. If we take $\forall F \subseteq N, \exists i \in F$ such that $\sum_{j \in F} a_{i j}>0$ from the premise. Hence, $\exists i \in F$ such that $\rho-\sum_{j \in F} a_{i j}<\rho$. Note that $\sum_{j \in F} b_{i j}=\rho-\sum_{j \in F} a_{i j}$ is true from the definition of $B$. Thus, $\exists i \in F$ such that $\sum_{j \in F} b_{i j}<\rho$ for $\forall F \subseteq N$. [Q. E. D.]

Then, we classify elements belonging to the number set $N$.

According to Lemma 2.8, $B$ itself, which is one of the principal submatrices of $B$, has at least one row sum which is less than $\rho$. We choose $\forall i \in N$ which satisfy $\sum_{j \in F} b_{i j}<\rho$ to belong to the set $H_{1} . H_{1} \neq \phi$ from the premise.

If $\forall i \in N$ belong to $H_{1}$, the classification is complete. In the following, we consider the case where $\exists i \in N$ which does not belong to $H_{1}$. First, we prove the following Lemma.

Lemma 2.9 $\sum_{j \in N} b_{i j}=\rho$ for $\forall i \notin H_{1}$.

Proof. By Lemma 2.7, $\sum_{j \in N} b_{i j} \leq \rho$ for $\forall i \in N$. Since $i \notin H_{1}, \sum_{j \in N} b_{i j}<\rho$ is not true from the definition of $H_{1}$. Thus, $\sum_{j \in N} b_{i j}=\rho$ for $\forall i \notin H_{1}$. [Q. E. D.]

We now consider the classification where $\exists i \in N$ that does not belong to $H_{1}$. We define $F$ as the complement of $H_{1}$. By Lemma 2.8, $\exists i \in F$ such that $\sum_{j \in F} b_{i j}<\rho$. We classify such $i$ as belonging to the set $\mathrm{H}_{2}$.

If $\forall i \in N$ belong to $H_{1}$ or $H_{2}$, the classification is complete. If $\exists i \in N$ which belongs to neither set, we 
execute the third classification.

Generally, $r$ classification steps are executed when $\exists i \in N$ which does not belong to any of $H_{1}, \cdots, H_{r-1}$. In such a case, we define $F$ as the complement of $\bigcup_{s=1}^{r-1} H_{s}$. Then, $\exists i \in F$ such that $\sum_{j \in N} b_{i j}<\rho$ by Lemma 2.8. We classify such $i$ as belonging to the set $H_{r}$. The next Lemma is obvious from the past consideration.

Lemma 2.10 $H_{r} \neq \phi$ if $\exists i \in N$ that belongs to the complement of $\bigcup_{s=1}^{r-1} H_{s}$.

Then, the following Lemmas are derived.

Lemma 2.11 If $H_{r} \neq \phi$ for $r \geq 2$, then $H_{s} \neq \phi$ for $1 \leq s \leq r-1$.

Proof. It is obvious by the method to construct $H_{r}$ and mathematical induction. [Q. E. D.]

Lemma 2.12 If $r \neq s$, then $H_{r} \cap H_{s}=\phi$.

Proof. Without loss of generality, we suppose $r>s$ and prove the Lemma under this supposition. If

$i \in H_{r}, i$ belongs to the complement of $\bigcup_{s=1}^{r-1} H_{s}$ by the definition of $H_{r}$. Hence, $i$ does not belong to $H_{s}$ where $r>s$. That is, $H_{r} \cap H_{s}=\phi$. [Q. E. D.]

From Lemmas 2.11 and 2.12, $H_{r}$ can be defined at most by $r=n$. In short, the classification is finished in limited time. If it is finished within $m$ times, we derive the next Lemmas.

Lemma 2.13 $N=\bigcup_{r=1}^{m} H_{r}$.

Proof. It is obvious that $N=\bigcup_{r=1}^{m} H_{r}$. Hence, we prove $N \subseteq \bigcup_{r=1}^{m} H_{r}$. We suppose that $\exists i \in N$ that belongs to the complement of $\bigcup_{r=1}^{m} H_{r}$. Then, $\exists i \in H_{m+1}$ from Lemma 2.10, but this contradicts the definition of $m$. By reductio ad absurdum, $\forall i \in N$ belong to $\bigcup_{r=1}^{m} H_{r}$. [Q. E. D.]

Lemma 2.14 We define $G=\bigcup_{s=1}^{r-1} H_{s}$. If $H_{r}$ where $r \geq 2$ is not empty, $\sum_{j \in G} b_{i j}>0$ for $\forall i \in H_{r}$.

Proof. Let $F$ be the complement of $G=\bigcup_{s=1}^{r-1} H_{s}$ and $i$ be any element of $H_{r}$ where $r \geq 2$. By Lemma 2.9, $\quad \sum_{j \in N} b_{i j}=\sum_{j \in F} b_{i j}+\sum_{j \in G} b_{i j}=\rho$ holds. Then, $\quad \sum_{j \in F} b_{i j}=\rho-\sum_{j \in G} b_{i j} \quad$ is satisfied. $\sum_{j \in F} b_{i j}<\rho$ holds by the definition of $H_{r}$. Therefore, $\rho-\sum_{j \in G} b_{i j}<\rho$ holds. Thus, we obtain $\sum_{j \in G} b_{i j}>0$. [Q. E. D.]

Note that because $B$ is a non-negative matrix, a non-negative eigenvector corresponding to $\lambda(B)$ exists by Theorem 1.3. However, $\lambda(B)$ refers to a maximum non-negative eigenvalue of $B$. Let $x_{j}$ be the $j$ th element of the non-negative eigenvector and $x_{k}$ be a maximum of $x_{j}$.

Lemma 2.15 If $k \in H_{1}, \lambda(B)<\rho$.

Proof. $x_{j} \leq x_{k}$ for $\forall j \in N$ from the definition of $k$. Further, $\forall b_{k j} \geq 0$ because $B$ is a non-negative matrix. From these two conditions, $b_{k j} x_{j} \leq b_{k j} x_{k}$ for $\forall j \in N$.

On the other hand, $\lambda(B) x_{k}=\sum_{j \in N} b_{k j} x_{j}$ is true from the definition of eigenvalue and eigenvector. From these, $\lambda(B) x_{k} \leq\left(\sum_{j \in N} b_{k j}\right) x_{k}$ is derived.

Note that if $x_{k}$, which is a maximum of the non-negative eigenvector, is zero, the eigenvector must be a zero vector. However, this contradicts the definition of eigenvector. Thus, $x_{k}>0$. Then if we divide both sides of the former formula by $x_{k}$, we derive $\lambda(B) \leq \sum_{j \in N} b_{k j}$. Note that $\sum_{j \in N} b_{k j}<\rho$ from the premise $k \in H_{1}$. From these two formulas, $\lambda(B)<\rho$ is derived. [Q. E. D.]

Lemma 2.16 Let $r$ be a natural number equal to or more than 2. If $k \notin \bigcup_{s=1}^{r-1} H_{s}$ and $k \in H_{r}$, then $\lambda(B)<\rho$.

Proof. We define $G=\bigcup_{s=1}^{r-1} H_{s}$ and $F$ as the complement of $G . F \neq \phi$ since $k \in H_{r}$, and $G=\bigcup_{s=1}^{r-1} H_{s} \neq \phi$ by Lemma 2.11. Let $h \in G$ such that $x_{j} \leq x_{h}$ for $\forall j \in G$. As $x_{j}$ is an element of the eigenvector corresponding to eigenvalue $\lambda(B), \lambda(B) x_{k}=\sum_{j \in F} b_{k j} x_{j}+\sum_{j \in G} b_{k j} x_{j}$ holds. $x_{j} \leq x_{h}$ for $\forall j \in G$ 
from the definition of $h$ and $b_{k j} \geq 0$ because $B$ is a non-negative matrix. Hence, $b_{k j} x_{j} \leq b_{k j} x_{h}$ for $\forall j \in G$ holds. Therefore, $\sum_{j \in G} b_{k j} x_{j} \leq\left(\sum_{j \in G} b_{k j}\right) x_{h}$ is satisfied. Further, $x_{j} \leq x_{k}$ by the definition of $k$ and $b_{k j} \geq 0$. Accordingly, $b_{k j} x_{j} \leq b_{k j} x_{k}$ for $\forall j \in F$ holds. Hence, $\sum_{j \in F} b_{k j} x_{j} \leq\left(\sum_{j \in F} b_{k j}\right) x_{k}$ is satisfied. From the above results, we see that $\lambda(B) x_{k}=\sum_{j \in F} b_{k j} x_{j}+\sum_{j \in G} b_{k j} x_{j} \leq\left(\sum_{j \in F} b_{k j}\right) x_{k}+\left(\sum_{j \in G} b_{k j}\right) x_{h}$.

We divide the leftmost and rightmost sides of this formula by $x_{k}>0, \lambda(B) \leq \sum_{j \in F} b_{k j}+\left(\sum_{j \in F} b_{k j}\right)\left(x_{h} / x_{k}\right)$ is derived. Note that $x_{k}$ is defined as the maximum of the elements in the non-negative eigenvector corresponding to $\lambda(B)$. Moreover, $k$ does not belong to $G$ and $h$ belongs to $G$. Hence, $x_{h}<x_{k}$. Therefore, $x_{h} / x_{k}<1$. From the premise $k \in H_{r}$ where $r \geq 2$ and Lemma 2.14, $\sum_{j \in G} b_{k j}>0$. Accordingly,

$\left(\sum_{j \in G} b_{k j}\right)\left(x_{h} / x_{k}\right)<\sum_{j \in G} b_{k j} \quad$ holds. Hence, $\lambda(B) \leq\left(\sum_{j \in F} b_{k j}\right)+\left(\sum_{j \in G} b_{k j}\right)\left(x_{h} / x_{k}\right)<\sum_{j \in F} b_{k j}+\sum_{j \in G} b_{k j}$ holds. As $\sum_{j \in N} b_{k j}=\sum_{j \in F} b_{k j}+\sum_{j \in G} b_{k j}$, we derive $\lambda(B)<\sum_{j \in N} b_{k j}$. Note that as $k \notin \bigcup_{s=1}^{r-1} H_{s}$ by the premise, it does not belong to $H_{1}$ either. Therefore, $\sum_{j \in N} b_{k j}=\rho$ by Lemma 2.9. $\lambda(B)<\sum_{j \in N} b_{k j}=\rho$ is derived. [Q. E. D.]

Proof of Theorem 2.6. By Lemma 2.13, $\forall k \in N$ belong to any $H_{r}$. By Lemmas 2.15 and 2.16, $\lambda(B)<\rho$ is true when $k$ belongs to any $H_{r}$. From Theorem 1.4, $A$ is a non-singular M-matrix. [Q. E. D.]

Now, we can show a necessary and sufficient condition for non-singularity of the NSZ-matrix. We will also show a necessary and sufficient condition for singularity of the matrix.

Theorem 2.17 A necessary and sufficient condition for non-singularity of the Z-matrix whose row sums are all non-negative is that at least one positive row sum exists in any principal submatrix of the matrix.

Proof. Necessity is shown in Theorem 2.5. Sufficiency is derived from Theorem 2.6. [Q. E. D.]

Theorem 2.18 A necessary and sufficient condition for singularity of the Z-matrix whose row sums are all non-negative is that there exists at least one principal submatrix of the matrix whose row sums are all zeroes.

Proof. By the contraposition of Theorem 2.17, a necessary and sufficient condition for singularity of the NSZ-matrix is that at least one principal submatrix of the NSZ-matrix whose row sums are all non-positive exists. From Theorem 2.3, this means that there exists at least one principal submatrix of the NSZ-matrix whose row sums are all zeroes. [Q. E. D.]

\section{A Necessary and Sufficient Condition for Non-Singularity of the Z-Matrix Which Has a Non-Negative Product with a Positive Vector}

In this section, we discuss the non-singularity of the NPZ-matrix. We reconfirm that the NPZ-matrix is defined as the Z-matrix $A$ which satisfies $A x \geq 0$ where $\exists x>0 . A=\left(a_{i j}\right)$ denotes an NPZ-matrix in this section.

We construct the diagonal matrix $P$ whose $i$ th diagonal element is the $i$ th element of $x$. As the diagonal elements of $P$ are all positive, its inverse $P^{-1}$ exists. Note that $P^{-1}$ is a diagonal matrix whose ith diagonal element is $1 / x_{i}$.

We subsequently construct a matrix $V=\left(v_{i j}\right)$ which satisfies $V=P^{-1} A P$. A and $V$ are similar to each other by the definition of matrix similarity.

Theorem $3.1 v_{i j}=a_{i j} x_{j} / x_{i}$ for $\forall i, j \in N$.

Proof. As the ith row vector of $P^{-1}$ is $\left(0, \cdots, 0,1 / x_{i}, 0, \cdots, 0\right)$ and the $j$ th column vector of $A$ is $\left(a_{1 j}, \cdots, a_{i-1 j}, a_{i j}, a_{i+1 j}, \cdots, a_{n j}\right)$, the $(i, j)$ th element of $P^{-1} A$ is $\left(1 / x_{i}\right) a_{i j}+\sum_{k \neq i} 0 a_{k j}=a_{i j} / x_{i}$. Thus, the $i$ th row vector of $P^{-1} A$ is $\left(a_{i 1} / x_{i}, \cdots, a_{i j-1} / x_{i}, a_{i j} / x_{i}, a_{i j+1} / x_{i}, \cdots, a_{i n} / x_{i}\right)$. Further, the $j$ th column vector of $P$ is $\left(0, \cdots, 0, x_{j}, 0, \cdots, 0\right)$. Thus, the $(i, j)$ th element of $V=P^{-1} A P$ is $v_{i j}=\left(a_{i j} / x_{i}\right) x_{j}+\sum_{h \neq j}\left(a_{i h} / x_{i}\right) 0=a_{i j} x_{j} / x_{i}$.

[Q. E. D.]

Theorem $3.2 V$ is an NSZ-matrix.

Proof. $a_{i j} \leq 0$ for $\forall i \neq j$ because $A$ is a Z-matrix, and $x_{i}>0, x_{j}>0$ because $x>0$. Therefore, $a_{i j} x_{j} / x_{i} \leq 0$ for $\forall i \neq j$. Then, $v_{i j} \leq 0$ for $\forall i \neq j$ by Theorem 3.1. That is, $V$ is a Z-matrix. 
Moreover, $\sum_{j \in N} a_{i j} x_{j} \geq 0$ for $\forall i \in N$ because $A$ is an NPZ-matrix. If we divide both sides of this formula by $x_{i}>0$, we obtain $\sum_{j \in N} a_{i j} x_{j} / x_{i} \geq 0$ for $\forall i \in N$. From Theorem 3.1, $\sum_{j \in N} v_{i j} \geq 0$ for $\forall i \in N$ is derived.

We have shown that $V$ is a Z-matrix and all row sums of $V$ are non-negative. Thus, $V$ satisfies the definition of an NSZ-matrix. [Q. E. D.]

Theorem 3.3 An NPZ-matrix $A$ is non-singular if and only if at least one positive row sum exists in any principal submatrix of $V$.

Proof. $V$ is an NSZ-matrix from Theorem 3.2. Then, by Theorem 2.17, $V$ is non-singular if and only if at least one positive row sum exists in any principal submatrix of $V$. Since $A$ and $V$ are similar to each other, this is also a necessary and sufficient condition for the non-singularity of $A$ by Theorem 1.6. [Q. E. D.]

Further, we will prove that this is also an equivalent condition that $A$ is a non-singular M-matrix.

Theorem 3.4 Take a real number $\rho$ which is equal to or more than all diagonal elements of $A$ and construct the matrix $B=\rho E-A$. Moreover, we construct $W=\rho E-V$. Then, $B$ and $W$ are similar to each other.

Proof. $P^{-1} B P=P^{-1}(\rho E-A) P=\rho P^{-1} E P-P^{-1} A P=\rho E-V=W$. [Q. E. D.]

Theorem 3.5 $W=\rho E-V$ is a non-negative matrix.

Proof. $\forall a_{i i} \leq \rho$ by the definition of $\rho$ and $v_{i i}=a_{i i} x_{i} / x_{i}=a_{i i}$ from Theorem 3.1. Then, $\forall v_{i i} \leq \rho$. Therefore, $\rho-v_{i i}$, which are diagonal elements of $W=\rho E-V$, are non-negative for $\forall i \in N$. Then, $-v_{i j}$, which are non-diagonal elements of $W=\rho E-V$, are non-negative for $\forall i \neq j$ because $V$ is a Z-matrix by Theorem 3.2. Thus, $W$ is a non-negative matrix. [Q. E. D.]

Theorem 3.6 An NPZ-matrix is an M-matrix .

Proof. $B=\rho E-A$ and $W=\rho E-V$ are both non-negative matrices due to Theorems 1.2 and 3.5. Thus, they have maximums of non-negative eigenvalues both by Theorem 1.3. Let $\lambda(B)$ and $\lambda(W)$ each be a maximum of a non-negative eigenvalue. Because $B$ and $W$ are similar by Theorem 3.4, $\lambda(B)=\lambda(W)$ by Theorem 1.7. Note that $V$ is an NSZ-matrix from Theorem 3.2. Then, $V$ is an M-matrix from Theorem 2.1. Therefore, $\lambda(W) \leq \rho$ from the definition of an M-matrix. Then, $\lambda(B) \leq \rho$ because $\lambda(B)=\lambda(W)$. We can confirm that $A$ satisfies the definition of an M-matrix. [Q. E. D.]

Theorem 3.7 An NPZ-matrix is non-singular if and only if it is a non-singular M-matrix.

Proof. It is obvious that an NPZ-matrix is non-singular if it is a non-singular M-matrix. Conversely, if an NPZ-matrix is non-singular, it is a non-singular M-matrix by Theorem 3.6. [Q. E. D.]

By Theorem 3.3, we can find a necessary and sufficient condition for non-singularity of an NPZ-matrix. However, this condition is described with parts of $V$ which is similar to $A$. It is not described with parts of $A$ and $x$ that are used in the definition of the NPZ-matrix. We will look for a condition described with such parts.

In the following, let $F$ be a subset of $N$ that is not empty, and $G$ be the complement of $F$ if $F$ is a proper subset. Then, the next Theorems hold.

Theorem 3.8 $\sum_{j \in F} v_{i j}>0$ if and only if $\sum_{j \in F} a_{i j} x_{j}>0$ for $\forall i \in F$ where $\forall F \subseteq N$.

Proof. This is true because $\sum_{j \in F} v_{i j}=\left(\sum_{j \in F} a_{i j} x_{j}\right) / x_{i}$ from Theorem 3.1 and $\forall x_{i}>0$ because $x$ is a positive vector. [Q. E. D.]

Theorem 3.9 If $F$ is a proper subset, $-a_{i j} x_{j} \geq 0$ for $\forall i \in F, \forall j \in G$.

Proof. Since $G$ is the complement of $F, a_{i j}$ for $\forall i \in F, \forall j \in G$ are non-diagonal elements of $A$. Then, they are non-positive because $A$ is a Z-matrix. Moreover, $\forall x_{j}>0$ because $x$ is a positive vector. Thus, $-a_{i j} x_{j} \geq 0$ for $\forall i \in F, \forall j \in G$. [Q. E. D.]

Theorem 3.10 $\sum_{j \in F} a_{i j} x_{j} \geq 0$ for $\forall i \in F$ where $\forall F \subseteq N$.

Proof. If $F=N$, this is obvious because of the definition of the NPZ-matrix. In the following, we show a proof for $F \subsetneq N$.

By the definition of an NPZ-matrix, $\sum_{j \in N} a_{i j} x_{j}=\sum_{j \in F} a_{i j} x_{j}+\sum_{j \in G} a_{i j} x_{j} \geq 0$ is satisfied. Hence, we obtain $\sum_{j \in F} a_{i j} x_{j} \geq-\sum_{j \in G} a_{i j} x_{j}$. Note that $-\sum_{j \in G} a_{i j} x_{j} \geq 0$ for $\forall i \in F$ holds from Theorem 3.9. Accordingly, $\sum_{j \in F} a_{i j} x_{j} \geq-\sum_{j \in G} a_{i j} x_{j} \geq 0$. [Q. E. D.] 
Theorem 3.11 Let $i$ be any element of $\forall F \subseteq N$. Then, $\sum_{j \in N} a_{i j} X_{j}>0$ or $\exists j \in G$ such that $a_{i j}<0$ is a necessary and sufficient condition for $\sum_{j \in F} a_{i j} x_{j}>0$.

\section{Proof.}

[Sufficiency] We prove this Theorem by dividing it into two cases.

(1) The case $\sum_{j \in N} a_{i j} x_{j}>0$.

If $F=N$, this is obvious. In the following, we show a proof for $F \subsetneq N$.

By the supposition, $\sum_{j \in N} a_{i j} x_{j}=\sum_{j \in F} a_{i j} x_{j}+\sum_{j \in G} a_{i j} x_{j}>0$ is satisfied. Therefore, we obtain

$\sum_{j \in F} a_{i j} x_{j}>-\sum_{j \in G} a_{i j} x_{j}$. Note that if $i \in F,-\sum_{j \in G} a_{i j} x_{j} \geq 0$ from Theorem 3.9. Hence,

$\sum_{j \in F} a_{i j} x_{j}>-\sum_{j \in G} a_{i j} x_{j} \geq 0$.

(2) The case $\exists j \in G$ such that $a_{i j}<0$.

If $F=N, G$ cannot be defined. Thus, this case is applied only when $F \subsetneq N$.

Let $k$ be one of $j \in G$ which satisfies $a_{i j}<0$, and $H$ be a set which removes $k$ from $G$. By the define tion of an NPZ-matrix, $\sum_{j \in N} a_{i j} x_{j}=\sum_{j \in F} a_{i j} x_{j}+\sum_{j \in H} a_{i j} x_{j}+a_{i k} x_{k} \geq 0$ is satisfied. Then we obtain $\sum_{j \in F} a_{i j} x_{j}+\sum_{j \in H} a_{i j} x_{j} \geq-a_{i k} x_{k}$. Since we consider the case $a_{i k}<0$, and $x_{k}>0$ is true from the definition of an NPZ-matrix, we obtain $-a_{i k} x_{k}>0$. Then $\sum_{j \in F} a_{i j} x_{j}+\sum_{j \in H} a_{i j} x_{j}>0$; in other words $\sum_{j \in F} a_{i j} x_{j}>-\sum_{j \in H} a_{i j} x_{j}$ is true. Further, considering $i \in F$ and $H \subset G,-\sum_{j \in H} a_{i j} x_{j} \geq 0$ is satisfied by Theorem 3.9. Therefore, we obtain $\sum_{j \in F} a_{i j} x_{j}>-\sum_{j \in H} a_{i j} x_{j} \geq 0$. [Q. E. D.]

[Necessity] By the definition of an NPZ-matrix, $\sum_{j \in N} a_{i j} x_{j} \geq 0$ for $\forall i \in F$ and $a_{i j} \leq 0$ for $\forall i \in F, \forall j \in G$ holds. Thus, the negative proposition of " $\sum_{j \in N} a_{i j} x_{j}>0$ or $\exists j \in G$ such that $a_{i j}<0$ ” is " $\sum_{j \in N} a_{i j} x_{j}=0$ and $a_{i j}=0$ for $\forall j \in G$ ". Further, as $i \in F$, the negative proposition of " $\sum_{j \in F} a_{i j} x_{j}>0$ " is “ $\sum_{j \in F} a_{i j} x_{j}=0$ ” from Theorem 3.10. Therefore, the contraposition of this Theorem is as follows. Let $i$ be any element of $\forall F \subseteq N$. If $\sum_{j \in N} a_{i j} x_{j}=0$ and $a_{i j}=0$ for $\forall j \in G, \sum_{j \in F} a_{i j} x_{j}=0$.

When $F=N$, this is obvious. When $F \subsetneq N$, if the supposition of the contraposition is satisfied,

$$
0=\sum_{j \in N} a_{i j} x_{j}=\sum_{j \in F} a_{i j} x_{j}+\sum_{j \in G} a_{i j} x_{j}=\sum_{j \in F} a_{i j} x_{j}+\sum_{j \in G} 0 x_{j}=\sum_{j \in F} a_{i j} x_{j}
$$

is true for $\forall i \in F$ where $\forall F \subseteq N$. That is, the contraposition is true. [Q. E. D.]

Now, we can show a necessary and sufficient condition for non-singularity of the NPZ-matrix described with parts of $A$ and $x$. We reconfirm that the NPZ-matrix is defined as a Z-matrix which satisfies $A x \geq 0$ where $\exists x>0$. Let $F$ be a subset of the number set $N$ which is not empty, and $G$ be the complement of $F$.

Theorem 3.12 Let the Z-matrix $A=\left(a_{i j}\right)$ satisfy $A x \geq 0$ where $\exists x>0$. A necessary and sufficient condition for the non-singularity of $A$ is $\exists i \in F$ such that $\sum_{j \in N} a_{i j} x_{j}>0$ or $\exists i \in F, \exists j \in G$ such that $a_{i j}<0$ for $\forall F \subseteq N^{10}$.

Proof. By Theorem 3.3, an NPZ-matrix is non-singular if and only if $\exists i \in F$ such that $\sum_{j \in F} v_{i j}>0$ for $\forall F \subseteq N$. By referring to Theorems 3.8 and 3.11, this condition can be rewritten as $\exists i \in F$ such that $\sum_{j \in N} a_{i j} x_{j}>0$ or $\exists i \in F, \exists j \in G$ such that $a_{i j}<0$ for $\forall F \subseteq N$. [Q. E. D.]

Theorem 3.13 Let the Z-matrix $A=\left(a_{i j}\right)$ satisfy $A x \geq 0$ where $\exists x>0$. A necessary and sufficient condition for the singularity of $A$ is $\exists F \subseteq N$ such that $\sum_{j \in N} a_{i j} x_{j}=0$ for $\forall i \in F$ and $a_{i j}=0$ for

$\forall i \in F, \forall j \in G^{11}$.

\footnotetext{
${ }^{10}$ In the case $F=N$, this condition is merely $\exists i \in F$ such that $\sum_{j \in N} a_{i j} X_{j}>0$.

${ }^{11}$ In the case $F=N$, this condition is merely $\sum_{j \in N} a_{i j} x_{j}=0$ for $\forall i \in F$.
} 
Proof. By the contraposition of Theorem 3.12, an NPZ-matrix is singular if and only if $\exists F \subseteq N$ such that $\sum_{j \in N} a_{i j} x_{j} \leq 0$ for $\forall i \in F$ and $a_{i j} \geq 0$ for $\forall i \in F, \forall j \in G$. However, $\sum_{j \in N} a_{i j} x_{j} \geq 0$ for $\forall i \in F$ and $a_{i j} \leq 0$ for $\forall i \in F, \forall j \in G$ are satisfied by the definition of an NPZ-matrix. Therefore, this singularity condition means $\exists F \subseteq N$ such that $\sum_{j \in N} a_{i j} x_{j}=0$ for $\forall i \in F$ and $a_{i j}=0$ for $\forall i \in F, \forall j \in G$. [Q. E. D.]

\section{Derivation from the Conditions by Robert Beauwens and Michael Neumann}

In fact, Robert Beauwens has already shown a condition which resembles what is shown in Theorem 2.17 as a necessary and sufficient condition for non-singularity of the NSZ-matrix. It is as follows.

First, we define the necessary concept.

If an $n$-dimensional square matrix $A=\left(a_{i j}\right)$ satisfies $\left|a_{i i}\right| \geq \sum_{i \neq j}\left|a_{i j}\right|$ for $\forall i \in N, A$ is called diagonally dominant.

Then, if a diagonally dominant matrix $A=\left(a_{i j}\right)$ satisfies $\left|a_{i i}\right|>\sum_{j=1}^{i-1}\left|a_{i j}\right|$ for $\forall i \in N, A$ is called lower semi-strictly diagonally dominant.

In the following, a permutation of $A$ denotes $B=P A P^{T}$ by a permutation matrix $P$. Then, if $B$, a permutation of $A$, is lower semi-strictly diagonally dominant, $A$ is called semi-strictly diagonally dominant.

Beauwens showed the following Theorem.

Theorem 4.1 Let the Z-matrix A be diagonally dominant and have diagonal elements that are all non-negative. $A$ is a non-singular M-matrix if and only if it is semi-strictly diagonally dominant ${ }^{12}$.

If $A$ is a Z-matrix, $\left|a_{i j}\right|=-a_{i j}$ holds for all non-diagonal elements. If diagonal elements of $A$ are nonnegative, $\left|a_{i i}\right|=a_{i i}$ holds for all diagonal elements.

Thus, if $A$ is diagonally dominant and has diagonal elements that are all non-negative, all row sums of $A$ are non-negative. Conversely, if all row sums of the Z-matrix $A$ are non-negative, diagonal elements of it are all non-negative by Theorem 2.3, and it is obviously diagonally dominant.

Therefore, the matrix which Theorem 4.1 addresses is nothing but the NSZ-matrix defined in this paper. Further, if $A=\left(a_{i j}\right)$ satisfies the premise of Theorem 4.1, $\left|a_{i i}\right|>\sum_{j=1}^{i-1}\left|a_{i j}\right|$ can be rewritten as $\sum_{j=1}^{i} a_{i j}>0$. Hence, Theorem 4.1 can be rewritten as follows.

Theorem 4.2 The NSZ-matrix $A=\left(a_{i j}\right)$ is a non-singular M-matrix if and only if $A$ satisfies $\sum_{j=1}^{i} a_{i j}>0$ for $\forall i \in N$ or $B=\left(b_{i j}\right)$, a permutation of $A$, satisfies $\sum_{j=1}^{i} b_{i j}>0$ for $\forall i \in N$.

Considering Theorem 2.2, Theorem 4.2 can be also rewritten as follows.

Theorem 4.3 The NSZ-matrix $A=\left(a_{i j}\right)$ is non-singular if and only if $A$ satisfies $\sum_{j=1}^{i} a_{i j}>0$ for $\forall i \in N$ or $B=\left(b_{i j}\right)$, a permutation of $A$, satisfies $\sum_{j=1}^{i} b_{i j}>0$ for $\forall i \in N$.

The Beauwens condition shown in Theorem 4.3 is equivalent to the condition shown in Theorem 2.17. However, before we prove this, we introduce another Theorem of Beauwens.

Theorem 4.4 The Z-matrix $A=\left(a_{i j}\right)$ is a non-singular M-matrix if and only if there exists a vector $x>0$ such that $A x \geq 0$ and $\sum_{j=1}^{i} a_{i j} x_{j}>0$ for $\forall i \in N^{13}$.

Furthermore, Michael Neumann showed the next Theorem.

Theorem 4.5 Let $A=\left(a_{i j}\right)$ be a Z-matrix and $B=\left(b_{i j}\right)$ be a permutation of $A$. A is a non-singular Mmatrix if and only if there exists a vector $x>0$ such that $B x \geq 0$ and $\sum_{j=1}^{i} b_{i j} x_{j}>0$ for $\forall i \in N^{14}$.

The matrix which Theorems 4.4 and 4.5 address is nothing but the NPZ-matrix defined in this paper. Considering also Theorem 3.7, the following Theorem can be derived.

Theorem 4.6 The NPZ-matrix $A=\left(a_{i j}\right)$ is non-singular if and only if $A$ satisfies $\sum_{j=1}^{i} a_{i j} x_{j}>0$ for

\footnotetext{
${ }^{12}$ Cf. Beauwens [9] pp. 110-111.

${ }^{13}$ It is written in Plemmons [10] p. 181, p. 183 and Berman \& Plemmons [1] p. 136, p. 162 that this theorem was first shown in Beauwens [9].

${ }^{14}$ It is written in Berman \& Plemmons [1] p. 136, p. 162 that this theorem was first shown in Neumann [11].
} 
$\forall i \in N$ or $B=\left(b_{i j}\right)$, a permutation of $A$, satisfies $\sum_{j=1}^{i} b_{i j} x_{j}>0$ for $\forall i \in N$.

The condition for non-singularity of the NPZ-matrix shown in Theorem 3.12 is equivalent to the Beauwens-Neumann condition shown in Theorem 4.6. We now prove this.

Theorem 4.7 Let $A=\left(a_{i j}\right)$ be an NPZ-matrix, and $B=\left(b_{i j}\right)$ be a permutation of $A . \exists i \in F$ such that $\sum_{j \in F} a_{i j} x_{j}>0$ for $\forall F \subseteq N$ is a necessary and sufficient condition that $\sum_{j=1}^{i} a_{i j} x_{j}>0$ for $\forall i \in N$ or $\sum_{j=1}^{i} b_{i j} x_{j}>0$ for $\forall i \in N$.

Proof.

[Sufficiency] Based on the premise, $\exists i \in N$ such that $\sum_{j \in N} a_{i j} x_{j}>0$. If we permute this $i$ with $n$, $\sum_{j=1}^{n} b_{i j} x_{j}>0$ is satisfied. Next, let $F_{n-1}$ be a set which removes $i$ from $N$. Based on the premise, $\exists i \in F_{n-1}$ such that $\sum_{j \in F_{n-1}} a_{i j} x_{j}>0$. If we permute this $i$ with $n-1, \sum_{j=1}^{n-1} b_{i j} x_{j}>0$ is satisfied. After this, in the range of $1 \leq r \leq n-2$, let $F_{r}$ be a set which removes $i$ from $F_{r+1}$. Based on the premise, $\exists i \in F_{r}$ such that $\sum_{j \in F_{r}} a_{i j} x_{j}>0$. If we permute this $i$ with $r, \sum_{j=1}^{r} b_{i j} x_{j}>0$ is satisfied. If these steps are executed to $r=1, \sum_{j=1}^{i} b_{i j} x_{j}>0$ holds for $\forall i \in N$. [Q. E. D.]

[Necessity] $\sum_{j \in F} a_{i j} x_{j} \geq 0$ is guaranteed by Theorem 3.10. Then, the contraposition of the proposition is as follows. If $\exists F \subseteq N$ such that $\sum_{j \in F} a_{i j} x_{j}=0$ for $\forall i \in F$, then $\exists i \in N$ such that $\sum_{j=1}^{i} a_{i j} x_{j}=0$ and $\exists i \in N$ such that $\sum_{j=1}^{i} b_{i j} x_{j}=0$. We prove this contraposition.

Let $k$ be the maximum number of elements of $F$ such that $\sum_{j \in F} a_{i j} x_{j}=0$ for $\forall i \in F$. Naturally, $\sum_{j \in F} a_{k j} x_{j}=0$ holds. Then, we prove $\sum_{j=1}^{k} a_{k j} x_{j}=0$.

If the number of elements of $F$ is more than $k, k$ cannot be the maximum number of elements of $F$. Hence, there is no such possibility. Thus, if we define $K=\{1,2, \cdots, k\}, F \subseteq K$ holds.

Then, we prove $\sum_{j=1}^{k} a_{k j} x_{j}=0$ by dividing it into two cases.

(1) The case $F=K$.

In this case, $\sum_{j=1}^{k} a_{k j} x_{j}=\sum_{j \in K} a_{k j} x_{j}=\sum_{j \in F} a_{k j} x_{j}$ is true. Moreover, $\sum_{j \in F} a_{k j} x_{j}=0$ is true from the premise. Thus, $\sum_{j=1}^{k} a_{k j} x_{j}=0$.

(2) The case $F \subsetneq K$.

Let $H$ be the relative complement of $F$ in $K . \sum_{j=1}^{k} a_{k j} x_{j}=\sum_{j \in K} a_{k j} x_{j}=\sum_{j \in F} a_{k j} x_{j}+\sum_{j \in H} a_{k j} x_{j}$ is true. Since $\sum_{j \in F} a_{k j} x_{j}=0$ from the premise, $\sum_{j \in K} a_{k j} x_{j}=\sum_{j \in H} a_{k j} x_{j} \cdot \sum_{j \in K} a_{k j} x_{j} \geq 0$ because of Theorem 3.10. Therefore, $\sum_{j \in H} a_{k j} x_{j} \geq 0$. On the other hand, $a_{k j} \leq 0$ for $\forall j \in H$ because $k \in F, H \not \subset F$ and $A$ is a Z-matrix. Further, $\forall x_{j}>0$ by the definition of the NPZ-matrix. Thus, $\sum_{j \in H} a_{k j} x_{j} \leq 0$. In order for these conditions to be compatible, we must have $\sum_{j \in H} a_{k j} x_{j}=0$. Therefore, $\sum_{j=1}^{k} a_{k j} x_{j}=\sum_{j \in K} a_{k j} x_{j}=\sum_{j \in H} a_{k j} x_{j}=0$.

Then, $\sum_{j=1}^{k} a_{k j} x_{j}=0$ is proved in any case. Thus, we obtain $\exists i \in N$ such that $\sum_{j=1}^{i} a_{i j} x_{j}=0$.

Next, we consider $B=\left(b_{i j}\right)$, a permutation of $A$. Let $F^{\prime}$ be a permutated set of $F$. Since $\exists F \subseteq N$ such that $\sum_{j \in F} a_{i j} x_{j}=0$ for $\forall i \in F$ is premised on the contraposition, then $\exists F^{\prime} \subseteq N$ such that $\sum_{j \in F^{\prime}} b_{i j} x_{j}=0$ for $\forall i \in F^{\prime}$.

Let $k^{\prime}$ be the maximum number of elements of $F^{\prime}$. Then, we can also prove $\sum_{j=1}^{k^{\prime}} b_{k^{\prime} j} x_{j}=0$ similarly to 
the proof for $\sum_{j=1}^{k} a_{k j} x_{j}=0$. Thus, we also obtain $\exists i \in N$ such that $\sum_{j=1}^{i} b_{i j} x_{j}=0$. [Q. E. D.]

Theorem 4.8 Let $A=\left(a_{i j}\right)$ be an NPZ-matrix, and $B=\left(b_{i j}\right)$ be a permutation of $A . \exists i \in F$ such that $\sum_{j \in N} a_{i j} x_{j}>0$ or $\exists i \in F, \exists j \in G$ such that $a_{i j}<0$ for $\forall F \subseteq N$ if and only if $\sum_{j=1}^{i} a_{i j} x_{j}>0$ for $\forall i \in N$ or $\sum_{j=1}^{i} b_{i j} x_{j}>0$ for $\forall i \in N$.

Proof. This is derived from Theorems 3.11 and 4.7 immediately. [Q. E. D.]

Theorem 4.8 shows the equivalence between the two non-singularity conditions of the NPZ-matrix, the condition in Theorem 3.12 and the Beauwens-Neumann condition in Theorem 4.6. Theorem 3.12 is also derived from Theorems 4.6 and 4.8 .

The equivalence between the two non-singularity conditions of the NSZ-matrix, the condition in Theorem 2.17 and the Beauwens condition in Theorem 4.3, can be also proved.

Theorem 4.9 Let $A=\left(a_{i j}\right)$ be an NSZ-matrix, and $B=\left(b_{i j}\right)$ be a permutation of $A$. $\exists i \in F$ such that $\sum_{j \in F} a_{i j}>0$ for $\forall F \subseteq N$ if and only if $\sum_{j=1}^{i} a_{i j}>0$ for $\forall i \in N$ or $\sum_{j=1}^{i} b_{i j}>0$ for $\forall i \in N$.

Proof. By Theorem 1.1, $A$ is equivalent to an NPZ-matrix where all elements of $x$ are the same number $x^{*}$. Therefore, considering Theorem 4.7 in the case all $x_{j}$ are equal to $x^{*}, \exists i \in F$ such that

$\left(\sum_{j \in F} a_{i j}\right) x^{*}>0$ for $\forall F \subseteq N$ if and only if $\left(\sum_{j=1}^{i} a_{i j}\right) x^{*}>0$ for $\forall i \in N$ or $\left(\sum_{j=1}^{i} b_{i j}\right) x^{*}>0$ for $\forall i \in N$. If we divide $\left(\sum_{j \in F} a_{i j}\right) x^{*}>0$ and $\left(\sum_{j=1}^{i} a_{i j}\right) x^{*}>0$ and $\left(\sum_{j=1}^{i} b_{i j}\right) x^{*}>0$ by $x^{*}>0$, we obtain this Theorem. [Q. E. D.]

If $i \in F, \sum_{j \in F} a_{i j}$ means a row sum of a principal submatrix of $A$. Thus, $\exists i \in F$ such that $\sum_{j \in F} a_{i j}>0$ for $\forall F \subseteq N$ means that at least one positive row sum exists in any principal submatrix of $A=\left(a_{i j}\right)$. Hence, Theorem 2.17 can be also derived from Theorems 4.3 and 4.9 .

\section{References}

[1] Berman, A. and Plemmons, R.J. (1979) Nonnegative Matrices in the Mathematical Sciences. Academic Press, Cambridge.

[2] Ostrowski, A. (1937-38) Über die Determinanten mit überwiegender Hauptdiagonale. Commentarii Mathematici Helvetici, 10, 69-96. http://dx.doi.org/10.1007/BF01214284

[3] Varga, R.S. (2000) Matrix Iterative Analysis. 2nd Revised and Expanded Edition, Springer, Berlin.

[4] Nikaido, H. (1968) Convex Structures and Economic Theory. Academic Press, Cambridge.

[5] DeFranza, J. and Gabliardi, D. (2009) Introduction to Linear Algebra with Applications. International Edition, The McGrow-Hill Higher Education.

[6] Anton, H. and Rorres, C. (2011) Elementary Linear Algebra with Supplement Applications. International Student Version,10th Edition, John Wiley \& Sons, Boston.

[7] Bretscher, O. (2009) Linear Algebra with Applications. 4th Edition, Pearson Prentice Hall, Upper Saddle River.

[8] Plemmons, R.J. (1976) M-Matrices Leading to Semiconvergent Splittings. Linear Algebra and its Applications, 15, 243-252. http://dx.doi.org/10.1016/0024-3795(76)90030-6

[9] Beauwens, R. (1976) Semistrict Diagonal Dominance. SIAM Journal on Numerical Analysis, 13, 109-112. http://dx.doi.org/10.1137/0713013

[10] Plemmons, R.J. (1977) M-Matrix Characterizations. 1—Nonsingular M-Matrices. Linear Algebra and Its Applications, 18, 175-188. http://dx.doi.org/10.1016/0024-3795(77)90073-8

[11] Neumann, M. (1979) A Note on Generalizations of Strict Diagonal Dominance for Real Matrices. Linear Algebra and Its Applications, 26, 3-14. http://dx.doi.org/10.1016/0024-3795(79)90168-X 